\title{
MODIFIED SHEPHARD'S PROBLEM ON PROJECTIONS OF CONVEX BODIES
}

\author{
VLADYSLAV YASKIN
}

\begin{abstract}
We disprove a conjecture of A. Koldobsky asking whether it is enough to compare $(n-2)$-derivatives of the projection functions of two symmetric convex bodies in the Shephard problem in order to get a positive answer in all dimensions.
\end{abstract}

\section{INTRODUCTION}

Sections and projections of convex bodies have been actively studied for many years. Although their properties exhibit certain duality, there is no clear picture explaining this. The following two famous problems ask similar questions about sections and projection. Let $K$ and $L$ be origin-symmetric convex bodies in $\mathbb{R}^{n}$. The Busemann-Petty problem asks whether the assumption that all central hyperplane sections of $L$ have smaller volume than those $L$ implies that $K$ has smaller $n$-dimensional volume. Its counterpart for projections is known as the Shephard problem. It asks whether

$$
\operatorname{vol}_{n-1}\left(K \mid \theta^{\perp}\right) \leq \operatorname{vol}_{n-1}\left(L \mid \theta^{\perp}\right)
$$

for all $\theta \in S^{n-1}$ implies that

$$
\operatorname{vol}_{n}(K) \leq \operatorname{vol}_{n}(L)
$$

The latter problem was solved independently by Petty $[\mathrm{P}]$ and Schneider [S1], who showed that the implication is correct only if $n=2$. The solution to the Busemann-Petty problem was settled through the efforts of many people (for historical details see [K3, pp.3-7]) and it turned out that the answer to this problem is affirmative only in dimensions $n \leq 4$.

A unified approach to these problems was given by Koldobsky, Ryabogin and Zvavitch [KRZ1], [KRZ2, see also [K3, Section 5.1 and Section 8.4]. They showed that these two problems are essentially of the same nature, if treated with the help of Fourier analysis.

Koldobsky [K2] and Koldobsky, Yaskin, Yaskina [KYY] considered a modification of the Busemann-Petty problem, which gave a positive answer to the problem in all dimensions. Namely, for an origin-symmetric convex body

2000 Mathematics Subject Classification. 52A20, 52A38, 42B10.

Key words and phrases. Convex body, projection, Shephard's problem, Fourier transform. 
$K$ in $\mathbb{R}^{n}$, define the section function

$$
S_{K}(\xi)=\operatorname{vol}_{n-1}\left(K \cap \xi^{\perp}\right), \quad \xi \in S^{n-1},
$$

where $\xi^{\perp}$ is the central hyperplane in $\mathbb{R}^{n}$ orthogonal to $\xi$, and extend $S_{K}$ from the sphere to the whole $\mathbb{R}^{n}$ as a homogeneous function of degree -1 . Let $\Delta$ be the Laplace operator on $\mathbb{R}^{n}$. It was proved that for two originsymmetric infinitely smooth convex bodies $K, L$ in $\mathbb{R}^{n}$ and $\alpha \in \mathbb{R}, \alpha \geq n-4$, the condition

$$
(-\Delta)^{\alpha / 2} S_{K}(\xi) \leq(-\Delta)^{\alpha / 2} S_{L}(\xi), \quad \forall \xi \in S^{n-1}
$$

implies that $\operatorname{vol}_{n}(K) \leq \operatorname{vol}_{n}(L)$, while for $\alpha<n-4$ this is not necessarily true.

Koldobsky conjectured that a similar result must hold for projections, with $\alpha=n-2$ being the critical value after which the answer becomes affirmative. This conjecture was based on the following. First of all, for all origin-symmetric convex bodies $\|x\|_{K}^{-n+3}$ is a positive definite distribution, see [K3, Section 4.2]. In addition the authors of [KYY] showed that $\|x\|_{K}^{-1}|x|_{2}^{-n+4}$ is also positive definite, which corresponds to the borderline case of the result mentioned above. (In general $\|x\|_{K}^{-n+p+3} \cdot|x|_{2}^{-p}$ is positive definite for a certain range of $p$ ). One can see that all these functions have a common property: they are homogeneous of degree $-n+3$, and therefore it seemed plausible that $\|x\|_{K}|x|_{2}^{-n+2}$ should also be positive definite. (This would correspond to the case $p=n-2$ above). However in [KYY] the authors were unable to extend the proof to this case.

Here we prove that $\|x\|_{K}|x|_{2}^{-n+2}$ is not necessarily positive definite, giving a negative answer to the conjecture of Koldobsky. This seems to be one of not many examples where the direct analogy between sections and projections does not hold.

For other generalizations of the Shephard problem see [B], GZ], RZ].

Acknowledgments. The author would like to thank Professors Paul Goodey and Alexander Koldobsky for fruitful discussions.

\section{Convex Geometry and the Fourier transform}

The standard references here are the books by Gardner [G], Schneider [S2] and Koldobsky [K3]. Let $K$ be an origin-symmetric star body in $\mathbb{R}^{n}$. The Minkowski functional of $K$ is defined as

$$
\|x\|_{K}=\min \{a \geq 0: x \in a K\} .
$$

The function $\rho_{K}(x)=\|x\|_{K}^{-1}$ is called the radial function of $K$. If $x \in S^{n-1}$, $\rho_{K}(x)$ is the distance from the origin to the boundary of $K$ in the direction of $x$.

We say that a body $K$ is infinitely smooth if its radial function $\rho_{K}$ restricted to the unit sphere $S^{n-1}$ belongs to the space $C^{\infty}\left(S^{n-1}\right)$ of infinitely differentiable functions on the unit sphere. 
Throughout the paper we use the Fourier transform of distributions. The Fourier transform of a distribution $f$ is defined by $\langle\hat{f}, \phi\rangle=\langle f, \hat{\phi}\rangle$ for every test function $\phi$ from the Schwartz space $\mathcal{S}$ of rapidly decreasing infinitely differentiable functions on $\mathbb{R}^{n}$. For any even distribution $f$, we have $(\hat{f})^{\wedge}=$ $(2 \pi)^{n} f$.

In particular we are interested in the Fourier transform of homogeneous functions on $\mathbb{R}^{n}$. We will need the following version of Parseval's formula on the sphere proved by Koldobsky, see e.g. [K3, p.66].

Lemma 2.1. If $K$ and $L$ are origin-symmetric infinitely smooth star bodies in $\mathbb{R}^{n}$ and $0<p<n$, then $\left(\|x\|_{K}^{-p}\right)^{\wedge}$ and $\left(\|x\|_{L}^{-n+p}\right)^{\wedge}$ are continuous functions on $S^{n-1}$ and

$$
\int_{S^{n-1}}\left(\|x\|_{K}^{-p}\right)^{\wedge}(\xi)\left(\|x\|_{L}^{-n+p}\right)^{\wedge}(\xi) d \xi=(2 \pi)^{n} \int_{S^{n-1}}\|x\|_{K}^{-p}\|x\|_{L}^{-n+p} d x .
$$

Remark. The preceding lemma was formulated for Minkowski functionals, but in fact it holds true for arbitrary infinitely differentiable even functions on the sphere extended to $\mathbb{R}^{n} \backslash\{0\}$ as homogeneous functions of corresponding degrees.

We say that a distribution $f$ is positive definite if its Fourier transform is a positive distribution, in the sense that $\langle\hat{f}, \phi\rangle \geq 0$ for every non-negative test function $\phi$.

The next result from GKS will be our main tool in determining whether a homogeneous function represents a positive definite distribution, see also [K3, p.60].

Theorem 2.2. (Gardner, Koldobsky, Schlumprecht) Let $K$ be an infinitely smooth origin-symmetric star body in $\mathbb{R}^{n}$, and let $k \in \mathbb{N} \cup\{0\}, k \neq n-1$. Suppose that $\xi \in S^{n-1}$, and let $A_{K, \xi}$ be the corresponding parallel section function of $K: A_{K, \xi}(z)=\int_{K \cap(x, \xi)=z} d x$.

(a) If $q$ is not an integer, $-k-1<q<k$, then

$$
\begin{aligned}
& \left(\|x\|_{K}^{-n+q+1}\right)^{\wedge}(\xi)=\frac{\pi(n-q-1)}{\Gamma(-q) \cos \frac{\pi q}{2}} \times \\
& \quad \times \int_{0}^{\infty} \frac{A_{K, \xi}(z)-A_{\xi}(0)-A_{K, \xi}^{\prime \prime}(0) \frac{z^{2}}{2}-\cdots-A_{K, \xi}^{(k-1)}(0) \frac{z^{k-1}}{(k-1) !}}{z^{q+1}} d z .
\end{aligned}
$$

(b) If $k$ is an even integer, then

$$
\left(\|x\|_{K}^{-n+k+1}\right)^{\wedge}(\xi)=(-1)^{k / 2} \pi(n-k-1) A_{K, \xi}^{(k)}(0) .
$$

(c) If $k$ is an odd integer, then

$$
\begin{aligned}
& \left(\|x\|_{K}^{-n+k+1}\right)^{\wedge}(\xi)=(-1)^{(k+1) / 2} 2(n-1-k) k ! \times \\
& \quad \times \int_{0}^{\infty} \frac{A_{K, \xi}(z)-A_{K, \xi}(0)-A_{K, \xi}^{\prime \prime}(0) \frac{z^{2}}{2}-\cdots-A_{K, \xi}^{(k-1)}(0) \frac{z^{k-1}}{(k-1) !}}{z^{k+1}} d z .
\end{aligned}
$$


Here $A_{K, \xi}^{(k)}$ stands for the derivative of the order $k$ and the Fourier transform is considered in the sense of distributions.

Remarks. (i) The previous theorem implies that for infinitely smooth bodies the Fourier transform of $\|x\|^{-n+q+1}$ restricted to the unit sphere is a continuous function (see also [K3, Section 3.3]).

(ii) If $k=0$, then part (a) of the theorem reads as follows. For $-1<q<0$,

$$
\left(\|x\|_{K}^{-n+q+1}\right)^{\wedge}(\xi)=\frac{\pi(n-q-1)}{\Gamma(-q) \cos \frac{\pi q}{2}} \int_{S^{n-1}}|(\theta, \xi)|^{-q-1}\|\theta\|_{K}^{-n+q+1} d \theta .
$$

In particular, if $-1<q<0$, then $\left(\|x\|_{K}^{-n+q+1}\right)^{\wedge}$ is a non-negative function on the sphere for any star body $K$.

An extension of Theorem 2.2 to the case when $k=n-1$ was given in KKYY].

Theorem 2.3. Let $K$ be an infinitely smooth origin-symmetric star body in $\mathbb{R}^{n}$. Extend $A_{K, \xi}^{(n-1)}(0)$ to a homogeneous function of degree $-n$ of the variable $\xi \in \mathbb{R}^{n} \backslash\{0\}$. Then $\left(\ln \|\cdot\|_{K}\right)^{\wedge}$ is a continuous function on $\mathbb{R}^{n} \backslash\{0\}$ and

$$
A_{K, \xi}^{(n-1)}(0)=-\frac{\cos (\pi(n-1) / 2)}{\pi}\left(\ln \|\cdot\|_{K}\right)^{\wedge}(\xi),
$$

as distributions (of the variable $\xi$ ) acting on test functions with compact support outside of the origin. In particular,

i) if $n$ is odd

$$
\left(\ln \|x\|_{K}\right)^{\wedge}(\xi)=(-1)^{(n+1) / 2} \pi A_{K, \xi}^{(n-1)}(0), \quad \xi \in \mathbb{R}^{n} \backslash\{0\},
$$

ii) if $n$ is even, then for $\xi \in \mathbb{R}^{n} \backslash\{0\}$,

$$
\begin{aligned}
& \left(\ln \|x\|_{K}\right)^{\wedge}(\xi)= \\
& \quad=a_{n} \int_{0}^{\infty} \frac{A_{K, \xi}(z)-A_{K, \xi}(0)-A_{K, \xi}^{\prime \prime}(0) \frac{z^{2}}{2}-\ldots-A_{K, \xi}^{n-2}(z) \frac{z^{n-2}}{(n-2) !}}{z^{n}} d z,
\end{aligned}
$$

where $a_{n}=2(-1)^{n / 2+1}(n-1)$ !

The support function of a convex body $K$ in $\mathbb{R}^{n}$ is defined by

$$
h_{K}(x)=\max _{\xi \in K}(x, \xi), \quad x \in \mathbb{R}^{n} .
$$

If $K$ is origin-symmetric, then $h_{K}$ is the Minkowski norm of the polar body $K^{*}$.

Let $\operatorname{vol}_{n-1}\left(K \mid \theta^{\perp}\right)$ denote the $(n-1)$-dimensional volume of the orthogonal projection of the body $K$ onto the hyperplane orthogonal to $\theta$. The following is the well-known Cauchy formula [G, p. 361]:

$$
\operatorname{vol}_{n-1}\left(K \mid \theta^{\perp}\right)=\frac{1}{2} \int_{S^{n-1}}|(\xi, \theta)| d S_{n-1}(K, \xi),
$$


where $d S_{n-1}(K, \xi)$ is the surface area measure of $K([\mathrm{G}, \mathrm{p} .351])$. A convex body $K$ is said to have a curvature function $f_{K}$, if its surface area measure $d S_{n-1}(K, \xi)$ is absolutely continuous with respect to Lebesgue measure $d \sigma_{n-1}$ on $S^{n-1}$ and

$$
\frac{d S_{n-1}(K, \cdot)}{d \sigma}=f_{K}(\cdot) \in L^{1}\left(S^{n-1}\right)
$$

If $K$ is an infinitely smooth body with positive curvature, then $f_{K}(\theta)$ is the reciprocal of the Gauss curvature at the boundary point with unit normal $\theta$, see [S2, p. 419]. Abusing notations, we will also denote by $f_{K}$ the extension of $f_{K}$ to $\mathbb{R}^{n}$ as a homogeneous function of degree $-n-1$. Koldobsky, Ryabogin and Zvavitch [KRZ1] proved that if a body $K$ has a curvature function, then

$$
\operatorname{vol}_{n-1}\left(K \mid \theta^{\perp}\right)=-\frac{1}{\pi} \widehat{f_{K}}(\theta), \quad \forall \theta \in S^{n-1} .
$$

Let $\Delta$ be the Laplace operator on $\mathbb{R}^{n}$. The fractional powers of the Laplacian of a distribution $g$ are defined by

$$
(-\Delta)^{\alpha / 2} g=\frac{1}{(2 \pi)^{n}}\left(|x|_{2}^{\alpha} \hat{g}(x)\right)^{\wedge}
$$

where the Fourier transform is considered in the sense of distributions, and $|x|_{2}$ stands for the Euclidean norm in $\mathbb{R}^{n}$. Using the connection between the Fourier transform and differentiation, one can see that for an even integer $\alpha$ and an even distribution $g$ this definition gives the standard Laplacian applied $\alpha / 2$ times.

If $K$ is an infinitely smooth body with positive Gauss curvature, then $f_{K}$ is an infinitely differentiable function on the sphere (because the Gauss curvature is the determinant of the Weingarten map, which is infinitely differentiable and non-singular in our case, see [S2, pp.104-109]). Consider the projection function $\operatorname{vol}_{n-1}\left(K \mid(\cdot)^{\perp}\right)$ and extend it from the sphere to $\mathbb{R}^{n}$ as a homogeneous function of degree 1 . Using (3) and (4), we get

$$
(-\Delta)^{\alpha / 2} \operatorname{vol}_{n-1}\left(K \mid \theta^{\perp}\right)=-\frac{1}{\pi}\left(|x|_{2}^{\alpha} f_{K}(x)\right)^{\wedge}(\theta) .
$$

Since $|\cdot|_{2}^{\alpha} f_{K}$ is infinitely differentiable, $(-\Delta)^{\alpha / 2} \operatorname{vol}_{n-1}\left(K \mid(\cdot)^{\perp}\right)$ is a continuous function on the sphere ([K3, Lemma 3.16] or remark (i) after Theorem 2.2).

\section{MAin Results}

Let us start with a result in the positive direction.

Theorem 3.1. Let $n \leq \alpha<n+1$. Let $K, L \subset \mathbb{R}^{n}, n \geq 3$, be infinitely smooth origin-symmetric convex bodies with positive Gauss curvature such that

$$
(-\Delta)^{\alpha / 2} \operatorname{vol}_{n-1}\left(K \mid \theta^{\perp}\right) \geq(-\Delta)^{\alpha / 2} \operatorname{vol}_{n-1}\left(L \mid \theta^{\perp}\right), \quad \forall \theta \in S^{n-1} .
$$

Then $\operatorname{vol}_{n}(K) \leq \operatorname{vol}_{n}(L)$. 
Proof. Recall the following formula [G, p. 354]

$$
\operatorname{vol}_{n}(L)=\frac{1}{n} \int_{S^{n-1}} h_{L}(\theta) f_{L}(\theta) d \theta
$$

where $h_{L}$ and $f_{L}$ are the support function and curvature function of the body $L$ correspondingly. Therefore using the fact that $h_{L}=\|\cdot\|_{L^{*}}$ we get

$$
\begin{aligned}
\operatorname{vol}_{n}(L) & =\frac{1}{n} \int_{S^{n-1}}\|\theta\|_{L^{*}} f_{L}(\theta) d \theta \\
& =\frac{1}{n} \int_{S^{n-1}}|\theta|_{2}^{-\alpha}\|\theta\|_{L^{*}}|\theta|_{2}^{\alpha} f_{L}(\theta) d \theta .
\end{aligned}
$$

Since $n \leq \alpha<n+1$, remark (ii) after Theorem 2.2 implies that the Fourier transform of $|x|_{2}^{-\alpha}\|x\|_{L^{*}}$ is a non-negative function on the sphere. Applying the spherical version of Parseval's formula (Lemma 2.1), we get

$$
\begin{aligned}
& =\frac{1}{(2 \pi)^{n} n} \int_{S^{n-1}}\left(|x|_{2}^{-\alpha}\|x\|_{L^{*}}\right)^{\wedge}(\xi)\left(|x|_{2}^{\alpha} f_{L}(x)\right)^{\wedge}(\xi) d \xi \\
& =-\frac{\pi}{(2 \pi)^{n} n} \int_{S^{n-1}}\left(|x|_{2}^{-\alpha}\|x\|_{L^{*}}\right)^{\wedge}(\xi)(-\Delta)^{\alpha / 2} \operatorname{vol}_{n-1}\left(L \mid \xi^{\perp}\right) d \xi \\
& \geq-\frac{\pi}{(2 \pi)^{n} n} \int_{S^{n-1}}\left(|x|_{2}^{-\alpha}\|x\|_{L^{*}}\right)^{\wedge}(\xi)(-\Delta)^{\alpha / 2} \operatorname{vol}_{n-1}\left(K \mid \xi^{\perp}\right) d \xi \\
& =\frac{1}{n} \int_{S^{n-1}}\|\theta\|_{L^{*}} f_{K}(\theta) d \theta=\frac{1}{n} \int_{S^{n-1}} h_{L}(\theta) f_{K}(\theta) d \theta \\
& =V_{1}(K, L),
\end{aligned}
$$

where $V_{1}(K, L)$ is the mixed volume, also denoted by $V(K, \ldots, K, L)$, see $\mathrm{G}$, p.353], [S2, p.275].

Therefore we have $V_{1}(K, L) \leq \operatorname{vol}_{n}(L)$. Applying Minkowski's first inequality [S2, p. 317] we get

$$
\operatorname{vol}_{n}(L)^{\frac{1}{n}} \operatorname{vol}_{n}(K)^{\frac{n-1}{n}} \leq V_{1}(K, L) \leq \operatorname{vol}_{n}(L)
$$

and hence

$$
\operatorname{vol}_{n}(K) \leq \operatorname{vol}_{n}(L)
$$

Remark. Comparing the previous theorem with the original Shephard problem, one can observe that the inequality for the projections gets reversed. This happens because the answer to Shephard's problem is affirmative if $L$ is a polar projection body, that is the Fourier transform of $\|\cdot\|_{L^{*}}$ is a negative distribution outside of the origin, see [K3, pp.155-160]. On the other hand, as we have seen, if this norm is multiplied by the Euclidean norm to the appropriate power, then the Fourier transform of $|x|_{2}^{-\alpha}\|x\|_{L^{*}}$ becomes a positive distribution.

Lemma 3.2. Let $n-2 \leq \alpha<n, \alpha \neq 1$. Then there exists an originsymmetric convex body $L$ in $\mathbb{R}^{n}, n \geq 3$, such that $|x|_{2}^{-\alpha}\|x\|_{L}$ is not a positive definite distribution. 
Proof. First consider the case $n-2<\alpha<n$. For a large $N>0$ let $L$ be an ellipsoid with the norm:

$$
\|x\|_{L}=\left(x_{1}^{2}+\cdots+x_{n-1}^{2}+N x_{n}^{2}\right)^{1 / 2} .
$$

Define a star body $K \subset \mathbb{R}^{n}$ by the formula:

$$
\rho_{K}(\theta)=\rho_{L}^{\frac{1}{1-\alpha}}(\theta), \quad \theta \in S^{n-1},
$$

where $\rho_{K}$ and $\rho_{L}$ are the radial functions of the bodies $K$ and $L$ correspondingly. One can see that

$$
|x|_{2}^{-\alpha}\|x\|_{L}=\left(|x|_{2}^{-\alpha /(1-\alpha)}\|x\|_{L}^{1 /(1-\alpha)}\right)^{-\alpha+1}=\|x\|_{K}^{-\alpha+1}, \quad \forall x \in \mathbb{R}^{n} \backslash\{0\} .
$$

Using Theorem 2.2 with $q=n-\alpha \in(0,2)$ we get

$$
\left(\|x\|_{K}^{-\alpha+1}\right)^{\wedge}(\xi)=\frac{\pi(\alpha-1)}{\Gamma(\alpha-n) \cos \frac{\pi(n-\alpha)}{2}} \int_{0}^{\infty} t^{-n+\alpha-1}\left(A_{K, \xi}(t)-A_{K, \xi}(0)\right) d t,
$$

where the case $n-\alpha=1$ is understood in the sense of part (c) of the aforementioned theorem.

Note that $\Gamma(\alpha-n) \cos \frac{\pi(n-\alpha)}{2} \leq 0$ for $\alpha \in(n-2, n)$, where $\alpha=n-1$ is again understood in terms of the limit, so we need to prove that for some $\xi$

$$
\int_{0}^{\infty} t^{-n+\alpha-1}\left(A_{K, \xi}(t)-A_{K, \xi}(0)\right) d t>0 .
$$

Let $\xi$ be the direction of the $x_{n}$-axis. Let $\left[-t_{0}, t_{0}\right]$ be the support of $A_{K, \xi}(t)$, then

$$
\begin{gathered}
\int_{0}^{\infty} t^{-n+\alpha-1}\left(A_{K, \xi}(t)-A_{K, \xi}(0)\right) d t= \\
=\int_{0}^{t_{0}} t^{-n+\alpha-1}\left(A_{K, \xi}(t)-A_{K, \xi}(0)\right) d t-\int_{t_{0}}^{\infty} t^{-n+\alpha-1} A_{K, \xi}(0) d t \\
=\int_{0}^{t_{0}} t^{-n+\alpha-1}\left(A_{K, \xi}(t)-A_{K, \xi}(0)\right) d t-\frac{A_{K, \xi}(0)}{n-\alpha} t_{0}^{-n+\alpha} .
\end{gathered}
$$

Introduce the following coordinates on the sphere $S^{n-1}$. Every $\theta \in S^{n-1}$ can be written as

$$
\theta=\cos \phi \cdot \zeta+\sin \phi \cdot \xi
$$

where $-\pi / 2 \leq \phi \leq \pi / 2$ and $\zeta \in S^{n-1} \cap \xi^{\perp}$.

Since we are interested in the sections of $K$ perpendicular to $\xi$, its axis of revolution, by abuse of notation we will denote by $\rho_{K}(\phi)$ the radial function of those $\theta \in S^{n-1}$ that make an angle $\phi$ with the plane $\xi^{\perp}$. Explicitly it equals

$$
\rho_{K}(\phi)=\left(\cos ^{2} \phi+N \sin ^{2} \phi\right)^{1 /(2 \alpha-2)} .
$$

One can check that $t=\sin \phi \cdot \rho_{K}(\phi)$ is an increasing function of the angle $\phi \in(0, \pi / 2)$, therefore all the sections of $K$ by hyperplanes orthogonal to $\xi$ are $(n-1)$-dimensional disks. Moreover, one can see that $t_{0}=N^{\frac{1}{2 \alpha-2}}$, which implies that the last term in (7) approaches zero as $N$ tends to infinity. 
It will be more convenient to work with $\phi$ instead of $t . A_{K, \xi}$ as a function of $\phi$ looks as follows.

$$
\begin{aligned}
A_{K, \xi}(t(\phi)) & =\omega_{n-1}\left(\cos \phi \cdot \rho_{K}(\phi)\right)^{n-1} \\
& =\omega_{n-1}(\cos \phi)^{n-1}\left(\cos ^{2} \phi+N \sin ^{2} \phi\right)^{\frac{n-1}{2 \alpha-2}},
\end{aligned}
$$

where $\omega_{n-1}$ is the volume of the unit $(n-1)$-dimensional Euclidean ball.

Now consider the integral term from (7). Making change of the variable $t=\sin \phi \cdot\left(\cos ^{2} \phi+N \sin ^{2} \phi\right)^{1 /(2 \alpha-2)}$, we get

$$
\begin{gathered}
\int_{0}^{t_{0}} t^{-n+\alpha-1}\left(A_{K, \xi}(t)-A_{K, \xi}(0)\right) d t= \\
=\omega_{n-1} \int_{0}^{\pi / 2}(\sin \phi)^{-n+\alpha-1}\left(\cos ^{2} \phi+N \sin ^{2} \phi\right)^{\frac{-n+\alpha-1}{2 \alpha-2}} \times \\
\times\left((\cos \phi)^{n-1}\left(\cos ^{2} \phi+N \sin ^{2} \phi\right)^{\frac{n-1}{2 \alpha-2}}-1\right) \times \\
\times\left(\cos ^{2} \phi+N \sin ^{2} \phi\right)^{\frac{1}{2 \alpha-2}-1}\left(\cos ^{3} \phi+\left(N+\frac{N-1}{\alpha-1}\right) \cos \phi \sin ^{2} \phi\right) d \phi .
\end{gathered}
$$

Now we want to find the intervals where the integrand is positive or negative. So we need to solve the equation

$$
(\cos \phi)^{n-1}\left(\cos ^{2} \phi+N \sin ^{2} \phi\right)^{\frac{n-1}{2 \alpha-2}}-1=0,
$$

which is equivalent to

$$
(\cos \phi)^{2 \alpha}+N(\cos \phi)^{2 \alpha-2} \sin ^{2} \phi=1
$$

By showing that the function in the left hand side is first increasing and then decreasing to zero, one can see that the equation has two roots on the interval $[0, \pi / 2]$. One root is obvious: $\phi_{1}=0$. In order to determine the second root $\phi_{2}$, note that the maximum of the function in question is achieved when $\phi$ is roughly arccos $\sqrt{1-1 / \alpha}$, assuming $N$ is large. Therefore (9) together with the inequality $\phi_{2} \gtrsim \arccos \sqrt{1-1 / \alpha}$ gives

$$
N\left(\cos \phi_{2}\right)^{2 \alpha-2} \leq C(\alpha),
$$

and hence $\phi_{2}=\pi / 2-o\left(N^{\frac{1}{2-2 \alpha}}\right)$.

Now break the integral (8) into two parts according to where the integrand is positive or negative. It is negative on the interval $\left(\pi / 2-o\left(N^{\frac{1}{2-2 \alpha}}\right), \pi / 2\right)$ and one can easily show that the absolute value of the integral here is bounded above by

$$
C N^{\frac{\alpha-n-2}{2 \alpha-2}}
$$

which approaches zero as $N$ tends to infinity.

In order to estimate from below the positive part of the integral (8) it is enough to consider the interval $[\pi / 4, \pi / 3]$. One can check that when $N$ is large, the integral has order

$$
C N^{\frac{1}{2}}
$$

which approaches infinity as $N$ gets large. The inequality (맘) follows. 
Now consider $\alpha=n-2$. In this case Theorem 2.2 gives

$$
\left(\|x\|_{K}^{-\alpha+1}\right)^{\wedge}(\xi)=\pi(1-\alpha) A_{K, \xi}^{\prime \prime}(0)<0 .
$$

The latter inequality follows by direct computation.

The previous Lemma says nothing about the case when $\alpha=1$ (and therefore $n=3$ ). It may seem that the right analog would be to analyze the sign of $\left(|x|_{2}^{-1}\|x\|_{L}+\ln |x|_{2}\right)^{\wedge}$. But in fact, as one will see later, the following result is needed.

Lemma 3.3. There exists an origin-symmetric convex body $L$ in $\mathbb{R}^{3}$, such that the Fourier transform of

$$
|x|_{2}^{-1}\|x\|_{L}-\frac{\int_{S^{2}}\|\theta\|_{L} d \theta}{4 \pi\left(1+\Gamma^{\prime}(1)\right)} \ln |x|_{2}
$$

is not a positive distribution outside of the origin. Here $\Gamma^{\prime}$ is the derivative of the Gamma-function.

Proof. For $N>0$ large enough consider the following planar curve defined in polar coordinates by

$$
\rho(\phi)=\cos ^{N} \phi .
$$

Take only that part of the curve where the angle $\phi$ belongs to the interval

$$
\left[-\arcsin \frac{1}{\sqrt{N+1}}, \arcsin \frac{1}{\sqrt{N+1}}\right] \text {. }
$$

One can check that the end-points of the interval correspond to the extreme values of the $y$-coordinate (altitude). Rotate this arc around the $y$-axis and then attach two disks at the top and bottom to get a closed surface. Denote by $L$ the convex body bounded by this surface. Consider a star body $K$ given by the formula

$$
\|\theta\|_{K}=\exp \left(\|\theta\|_{L}\right), \quad \theta \in S^{2} .
$$

Therefore the radial function of $K$ equals

$$
\rho_{K}(\theta)=\exp \left(-\rho_{L}^{-1}(\theta)\right), \quad \theta \in S^{2} .
$$

One can also see that

$$
\ln \|x\|_{K}=|x|_{2}^{-1}\|x\|_{L}+\ln |x|_{2}, \quad x \in \mathbb{R}^{3} \backslash\{0\} .
$$

Let $\xi$ be the direction of the axis of revolution of $L$. Since $n=3$, by Theorem 2.3 we have

$$
\left(\ln \|x\|_{K}\right)^{\wedge}(\xi)=\pi A_{K, \xi}^{\prime \prime}(0) .
$$

(In fact this formula can only be applied if the body is smooth enough, but let us ignore this problem for a while and address it at the end of the proof).

If we denote by $\rho_{K}(\phi)$ the radial function of those $\theta \in S^{2}$ that make an angle $\phi$ with the plane $\xi^{\perp}$, then

$$
A_{K, \xi}(\phi)=\pi\left(\cos \phi \cdot \rho_{K}(\phi)\right)^{2}=\pi \cos ^{2} \phi \cdot \exp \left(-2 \rho_{L}^{-1}(\phi)\right) .
$$


Using that for small $\phi$ the function $\rho_{L}$ is given by formula (10) we get

$$
A_{K, \xi}^{\prime \prime}(0)=-2 \pi e^{-2}(N-1) \text {. }
$$

On the other hand from the construction of the body $L$ it follows that $L$ has smallest radius in the direction of $\xi$. Therefore for all $\theta \in S^{2}$,

$$
\rho_{L}(\theta) \geq \rho_{L}(\xi)=\left.\left(\cos ^{N} \phi \sin \phi\right)\right|_{\phi=\arcsin \frac{1}{\sqrt{N+1}}} \simeq \frac{C_{1}}{\sqrt{N+1}}
$$

which implies

$$
\int_{S^{2}}\|\theta\|_{L} d \theta \leq C_{2} \sqrt{N+1}
$$

for some constants $C_{1}, C_{2}>0$.

Also notice that part (i) of Theorem 2.3 gives

$$
\left(\ln |x|_{2}\right)^{\wedge}(\theta)=-2 \pi^{2}, \quad \forall \theta \in S^{2} .
$$

Therefore we have

$$
\begin{gathered}
\left(|x|_{2}^{-1}\|x\|_{L}-\frac{\int_{S^{2}}\|\theta\|_{L} d \theta}{4 \pi\left(1+\Gamma^{\prime}(1)\right)} \ln |x|_{2}\right)^{\wedge}(\xi) \\
=\left(\ln \|x\|_{K}-\frac{1+\Gamma^{\prime}(1)+\frac{1}{4 \pi} \int_{S^{2}}\|\theta\|_{L} d \theta}{1+\Gamma^{\prime}(1)} \ln |x|_{2}\right)^{\wedge}(\xi) \\
=-2 \pi e^{-2}(N-1)+2 \pi^{2} \frac{1+\Gamma^{\prime}(1)+\frac{1}{4 \pi} \int_{S^{2}}\|\theta\|_{L} d \theta}{1+\Gamma^{\prime}(1)} \\
\leq-2 \pi e^{-2}(N-1)+C \sqrt{N+1}<0,
\end{gathered}
$$

for $N>0$ large enough.

Formally the above computations are not quite legitimate since $L$ is not infinitely smooth. But one can approximate $L$ by an origin-symmetric infinitely smooth convex body without loosing the sign in the last inequality. Specifically, one has to smooth out the body in a small neighborhood of $\phi=\arcsin \frac{1}{\sqrt{N+1}}$. This operation will not affect (11). On the other hand one can also assure that $\int_{S^{2}}\|\theta\|_{L} d \theta$ does not change much.

Theorem 3.4. Let $n-2 \leq \alpha<n$. There are convex origin-symmetric bodies $K, L \subset \mathbb{R}^{n}, n \geq 3$ such that

$$
\begin{aligned}
(-\Delta)^{\alpha / 2} \operatorname{vol}_{n-1}\left(L \mid \theta^{\perp}\right) \leq & (-\Delta)^{\alpha / 2} \operatorname{vol}_{n-1}\left(K \mid \theta^{\perp}\right), \quad \forall \theta \in S^{n-1}, \\
\text { but } & \operatorname{vol}_{n}(L)<\operatorname{vol}_{n}(K) .
\end{aligned}
$$

Proof. First assume that $\alpha \neq 1$. Lemma 3.2 guarantees that there exists an ellipsoid $K^{*}$, such that $\left(|x|_{2}^{-\alpha}\|x\|_{K^{*}}\right)^{\wedge}(\xi)<0$ for some direction $\xi$. Let $K$ be the polar body of $K^{*}$. Since $K$ is again an ellipsoid, its curvature function $f_{K}$ is well-defined. 
Let $\Omega=\left\{\theta \in S^{n-1}:\left(|x|_{2}^{-\alpha}\|x\|_{K^{*}}\right)^{\wedge}(\theta)<0\right\}$ and let $v \in C^{\infty}\left(S^{n-1}\right)$ be a non-negative even function supported in $\Omega$. Extend $v$ to a homogeneous function $|x|_{2}^{1-\alpha} v\left(x /|x|_{2}\right)$ of degree $1-\alpha$ on $\mathbb{R}^{n}$. By [K1, Lemma 5] the Fourier transform of $|x|_{2}^{1-\alpha} v\left(x /|x|_{2}\right)$ is equal to $|x|_{2}^{-n-1+\alpha} g\left(x /|x|_{2}\right)$ for some function $g \in C^{\infty}\left(S^{n-1}\right)$. Choose an $\varepsilon>0$ small enough and define

$$
f_{L}(x)=f_{K}(x)+\varepsilon|x|_{2}^{-n-1} g\left(x /|x|_{2}\right)>0 .
$$

By Minkowski's existence theorem [G, p.356] there is a convex origin-symmetric body $L \in \mathbb{R}^{n}$ with such defined curvature function. Now multiply both sides by $|x|_{2}^{\alpha}$ and apply the Fourier transform to get

$$
\begin{aligned}
-\pi(-\Delta)^{\alpha / 2} \operatorname{vol}_{n-1}\left(L \mid \theta^{\perp}\right) & =-\pi(-\Delta)^{\alpha / 2} \operatorname{vol}_{n-1}\left(K \mid \theta^{\perp}\right)+(2 \pi)^{n} \varepsilon v(\theta) \\
& \geq-\pi(-\Delta)^{\alpha / 2} \operatorname{vol}_{n-1}\left(K \mid \theta^{\perp}\right) .
\end{aligned}
$$

On the other hand,

$$
\begin{gathered}
-\pi \int_{S^{n-1}}\left(|x|_{2}^{-\alpha}\|x\|_{K^{*}}\right)^{\wedge}(\theta)(-\Delta)^{\alpha / 2} \operatorname{vol}_{n-1}\left(L \mid \theta^{\perp}\right) d \theta= \\
=-\pi \int_{S^{n-1}}\left(|x|_{2}^{-\alpha}\|x\|_{K^{*}}\right)^{\wedge}(\theta)(-\Delta)^{\alpha / 2} \operatorname{vol}_{n-1}\left(K \mid \theta^{\perp}\right) d \theta+ \\
\quad+(2 \pi)^{n} \varepsilon \int_{S^{n-1}}\left(|x|_{2}^{-\alpha}\|x\|_{K^{*}}\right)^{\wedge}(\theta) v(\theta) d \theta< \\
-\pi \int_{S^{n-1}}\left(|x|_{2}^{-\alpha}\|x\|_{K^{*}}\right)^{\wedge}(\theta)(-\Delta)^{\alpha / 2} \operatorname{vol}_{n-1}\left(K \mid \theta^{\perp}\right) d \theta
\end{gathered}
$$

where the last inequality follows from the fact that $v$ is supported in the set, where $\left(|x|_{2}^{-\alpha}\|x\|_{K^{*}}\right)^{\wedge}<0$.

Using the argument from Theorem 3.1 we get that

$$
\operatorname{vol}_{n}(L)<\operatorname{vol}_{n}(K) .
$$

In order to prove the remaining case when $\alpha=1$, we need two Lemmas. The following Lemma is from [YY, Lemma 3.3], see also [KKYY].

Lemma 3.5. Let $K$ be an infinitely smooth origin-symmetric star body in $\mathbb{R}^{n}$. Then

$$
\ln \|x\|_{K}=-\frac{1}{(2 \pi)^{n}} \int_{S^{n-1}} \ln |(x, \xi)|\left(\ln \|x\|_{K}\right)^{\wedge}(\xi) d \xi+C_{K},
$$

where

$$
C_{K}=\frac{1}{\left|S^{n-1}\right|} \int_{S^{n-1}} \ln \|x\|_{K} d x-\frac{1}{2 \sqrt{\pi}} \Gamma^{\prime}(1 / 2)+\frac{1}{2} \frac{\Gamma^{\prime}(n / 2)}{\Gamma(n / 2)} .
$$

Moreover,

$$
\int_{S^{n-1}}\left(\ln \|x\|_{K}\right)^{\wedge}(\xi) d \xi=-(2 \pi)^{n} .
$$

The following result is from [YY, Lemma 3.7]. It is not stated in this form there, but follows from the proof. 
Lemma 3.6. Let $K$ be an origin-symmetric star body in $\mathbb{R}^{n}$, then the Fourier transform of $\|x\|_{K}^{-n}$ is a continuous function on $\mathbb{R}^{n} \backslash\{0\}$, which equals

$$
\left(\|x\|_{K}^{-n}\right)^{\wedge}(\xi)=\int_{S^{n-1}}\|\theta\|_{K}^{-n}\left(\Gamma^{\prime}(1)-\ln |(\theta, \xi)|\right) d \theta .
$$

Now we are able to prove the remaining case of the Theorem, when $\alpha=1$ (and therefore $n=3$ ). By Lemma 3.3 there exists an infinitely smooth origin-symmetric convex body $K^{*}$ in $\mathbb{R}^{3}$ such that for some $\xi \in S^{2}$

$$
\left(|x|_{2}^{-1}\|x\|_{K^{*}}-\frac{\int_{S^{2}}\|\theta\|_{K^{*}} d \theta}{4 \pi\left(1+\Gamma^{\prime}(1)\right)} \ln |x|_{2}\right)^{\wedge}(\xi)<0 .
$$

Let $K$ be the polar body of $K^{*}$. By approximation we can assume that $K$ is infinitely smooth with strictly positive curvature, see [S2, pp. 158-160].

Let

$$
\Omega=\left\{\theta \in S^{2}:\left(|x|_{2}^{-1}\|x\|_{K^{*}}-\frac{\int_{S^{2}}\|\theta\|_{K^{*}} d \theta}{4 \pi\left(1+\Gamma^{\prime}(1)\right)} \ln |x|_{2}\right)^{\wedge}(\theta)<0\right\}
$$

and let $v \in C^{\infty}\left(S^{2}\right)$ be an even function, $0<v \leq 1$, not identically equal to 1 , and such that $v=1$ in $S^{2} \backslash \Omega$. We will also use the fact that $\Gamma^{\prime}(1)>-1$ to impose an additional condition on $v$ :

$$
\frac{1}{4 \pi} \int_{S^{2}} \ln v(\theta) d \theta=-1-\Gamma^{\prime}(1)
$$

Note that the latter equality can be written in the form

$$
C_{v}+\Gamma^{\prime}(1)=0
$$

where $C_{v}$ is the constant from Lemma 3.5 .

Extend $v$ from the sphere to $\mathbb{R}^{3}$ as a homogeneous function of degree 1, and denote this extension also by $v$. By Theorem 2.3 the Fourier transform of $\ln v(x)$ outside of the origin is equal to $|x|_{2}^{-3} g\left(x /|x|_{2}\right)$ for some function $g \in C^{\infty}\left(S^{2}\right)$. Choose an $\varepsilon>0$ small enough and define

$$
f_{L}(x)=f_{K}(x)-\varepsilon|x|_{2}^{-4} g\left(x /|x|_{2}\right)>0 .
$$

By Minkowski's existence theorem there is a convex symmetric body $L \subset \mathbb{R}^{3}$ with such defined curvature function. Now multiply both sides by $|x|_{2}$ and apply the Fourier transform to get

$$
\begin{aligned}
-\pi(-\Delta)^{\alpha / 2} & \operatorname{vol}_{2}\left(L \mid \xi^{\perp}\right)= \\
& =-\pi(-\Delta)^{\alpha / 2} \operatorname{vol}_{2}\left(K \mid \xi^{\perp}\right)-(2 \pi)^{3} \varepsilon\left(|x|_{2}^{-3} g\left(x /|x|_{2}\right)\right)^{\wedge}(\xi) \\
& \geq-\pi(-\Delta)^{\alpha / 2} \operatorname{vol}_{2}\left(K \mid \xi^{\perp}\right),
\end{aligned}
$$

where the last inequality comes from the following calculations, based on Lemmas 3.6 and 3.5 .

$$
\left(|x|_{2}^{-3} g\left(x /|x|_{2}\right)\right)^{\wedge}(\xi)=\int_{S^{2}} g(\theta)\left(\Gamma^{\prime}(1)-\ln |(\theta, \xi)|\right) d \theta
$$




$$
\begin{gathered}
=\int_{S^{2}}(\ln v(x))^{\wedge}(\theta)\left(\Gamma^{\prime}(1)-\ln |(\theta, \xi)|\right) d \theta \\
=-(2 \pi)^{3} \Gamma^{\prime}(1)-\int_{S^{2}}(\ln v(x))^{\wedge}(\theta) \ln |(\theta, \xi)| d \theta \\
=-(2 \pi)^{3} \Gamma^{\prime}(1)+(2 \pi)^{3}\left(\ln v(\xi)-C_{v}\right)=(2 \pi)^{3} \ln v(\xi) \leq 0 .
\end{gathered}
$$

On the other hand

$$
\begin{gathered}
V_{1}(L, K)=\frac{1}{3} \int_{S^{2}}\|\theta\|_{K^{*}} f_{L}(\theta) d \theta=\frac{1}{3} \int_{S^{2}}\|\theta\|_{K^{*}}\left(f_{K}(\theta)-\varepsilon g(\theta)\right) d \theta \\
=\operatorname{vol}_{3}(K)-\frac{\varepsilon}{3} \int_{S^{2}}\|\theta\|_{K^{*}} g(\theta) d \theta
\end{gathered}
$$

If we can show that $\int_{S^{2}}\|\theta\|_{K^{*}} g(\theta) d \theta>0$, the statement will follow from Minkowski's first inequality. Let $\|\theta\|_{M}=\exp \|\theta\|_{K^{*}}$ for all $\theta \in S^{2}$. By Lemma 3.5 we have

$$
\|\theta\|_{K^{*}}=\ln \|\theta\|_{M}=-\frac{1}{(2 \pi)^{3}} \int_{S^{2}} \ln |(\theta, \xi)|\left(\ln \|x\|_{M}\right)^{\wedge}(\xi) d \xi+C_{M},
$$

where

$$
C_{M}=\frac{1}{4 \pi} \int_{S^{2}} \ln \|\theta\|_{M} d \theta+1=\frac{1}{4 \pi} \int_{S^{2}}\|\theta\|_{K^{*}} d \theta+1
$$

Analogously,

$$
\ln |\theta|_{2}=-\frac{1}{(2 \pi)^{3}} \int_{S^{2}} \ln |(\theta, \xi)|\left(\ln |x|_{2}\right)^{\wedge}(\xi) d \xi+1 .
$$

Let us denote

$$
\lambda=\frac{C_{M}+\Gamma^{\prime}(1)}{1+\Gamma^{\prime}(1)}
$$

Then

$$
\begin{gathered}
\int_{S^{2}}\|\theta\|_{K^{*}} g(\theta) d \theta=\int_{S^{2}}\left(\ln \|\theta\|_{M}-\lambda \ln |\theta|_{2}\right) g(\theta) d \theta= \\
=-\frac{1}{(2 \pi)^{3}} \int_{S^{2}}\left(\int_{S^{2}} \ln |(\theta, \xi)|\left(\ln \|x\|_{M}-\lambda \ln |x|_{2}\right)^{\wedge}(\xi) d \xi\right) g(\theta) d \theta \\
+\left(C_{M}-\lambda\right) \int_{S^{2}} g(\theta) d \theta .
\end{gathered}
$$

Reversing the order of integration in the first integral and then adding and subtracting an appropriate quantity, we get

$$
\begin{gathered}
=\frac{1}{(2 \pi)^{3}} \int_{S^{2}}\left(\int_{S^{2}}\left(\Gamma^{\prime}(1)-\ln |(\theta, \xi)|\right) g(\theta) d \theta\right)\left(\ln \|x\|_{M}-\lambda \ln |x|_{2}\right)^{\wedge}(\xi) d \xi \\
-\frac{\Gamma^{\prime}(1)}{(2 \pi)^{3}} \int_{S^{2}}\left(\int_{S^{2}} g(\theta) d \theta\right)\left(\ln \|x\|_{M}-\lambda \ln |x|_{2}\right)^{\wedge}(\xi) d \xi \\
+\left(C_{M}-\lambda\right) \int_{S^{2}} g(\theta) d \theta
\end{gathered}
$$


Formulas (15) and (14) applied to the first and second integrals correspondingly give

$$
\begin{gathered}
=\frac{1}{(2 \pi)^{3}} \int_{S^{2}}\left(|x|_{2}^{-3} g\left(x /|x|_{2}\right)\right)^{\wedge}(\xi)\left(\ln \|x\|_{M}-\lambda \ln |x|_{2}\right)^{\wedge}(\xi) d \xi \\
+\Gamma^{\prime}(1)(1-\lambda) \int_{S^{2}} g(\theta) d \theta+\left(C_{M}-\lambda\right) \int_{S^{2}} g(\theta) d \theta .
\end{gathered}
$$

Using that

$$
\left(|x|_{2}^{-3} g\left(x /|x|_{2}\right)\right)^{\wedge}(\xi)=(2 \pi)^{3} \ln v(\xi)
$$

and

$$
\Gamma^{\prime}(1)(1-\lambda)+\left(C_{M}-\lambda\right)=0
$$

we get

$$
\int_{S^{2}}\|\theta\|_{K^{*}} g(\theta) d \theta=\int_{S^{2}} \ln v(\xi)\left(\ln \|x\|_{M}-\lambda \ln |x|_{2}\right)^{\wedge}(\xi) d \xi .
$$

Recall that

$$
\begin{gathered}
\ln \|x\|_{M}-\lambda \ln |x|_{2} \\
=|x|_{2}^{-1}\|x\|_{K^{*}}+\ln |x|_{2}-\frac{\frac{1}{4 \pi} \int_{S^{2}}\|\theta\|_{K^{*}} d \theta+1+\Gamma^{\prime}(1)}{1+\Gamma^{\prime}(1)} \ln |x|_{2} \\
=|x|_{2}^{-1}\|x\|_{K^{*}}-\frac{\int_{S^{2}}\|\theta\|_{K^{*}} d \theta}{4 \pi\left(1+\Gamma^{\prime}(1)\right)} \ln |x|_{2} .
\end{gathered}
$$

Therefore (18) implies

$$
\int_{S^{2}}\|\theta\|_{K^{*}} g(\theta) d \theta>0
$$

since $\ln v$ is negative, where

$$
\left(|x|_{2}^{-1}\|x\|_{K^{*}}-\frac{\int_{S^{2}}\|\theta\|_{K^{*}} d \theta}{4 \pi\left(1+\Gamma^{\prime}(1)\right)} \ln |x|_{2}\right)^{\wedge}
$$

is negative, and zero everywhere else.

Remark. The aim of Theorem 3.4 is to show that condition (12) is inconclusive. As we have seen, there are bodies for which (12) holds, but $\operatorname{vol}_{n}(L)<\operatorname{vol}_{n}(K)$. Let us remark that one can easily find two bodies for which (12) holds, but $\operatorname{vol}_{n}(L)>\operatorname{vol}_{n}(K)$. It is enough to take two Euclidean balls. This is obvious for $\alpha>1$, but probably some explanations are needed for the case $\alpha=1$ (and $n=3$ ).

Let $B$ be a Euclidean ball in $\mathbb{R}^{3}$ with curvature function $f_{B}(\theta)=C$, $\forall \theta \in S^{2}$. Then by (5) and (15) we have

$$
\begin{aligned}
(-\Delta)^{1 / 2} \operatorname{vol}_{2}\left(B \mid \xi^{\perp}\right) & =-\frac{1}{\pi}\left(|x|_{2} f_{B}(x)\right)^{\wedge}(\theta)=-\frac{1}{\pi}\left(|x|_{2} C|x|_{2}^{-4}\right)^{\wedge}(\theta)= \\
= & -\frac{C}{\pi} \int_{S^{2}}\left(\Gamma^{\prime}(1)-\ln |(\theta, \xi)|\right) d \xi
\end{aligned}
$$


The latter integral is computable and after routine calculations one gets

$$
(-\Delta)^{1 / 2} \operatorname{vol}_{2}\left(B \mid \xi^{\perp}\right)=-4 C\left(\Gamma^{\prime}(1)+1\right)<0 .
$$

Therefore if we take two Euclidean balls $B_{r}$ and $B_{R}$ with radii $r<R$, then $f_{B_{r}}<f_{B_{R}}$ and therefore

$$
(-\Delta)^{1 / 2} \operatorname{vol}_{2}\left(B_{R} \mid \xi^{\perp}\right)<(-\Delta)^{1 / 2} \operatorname{vol}_{2}\left(B_{r} \mid \xi^{\perp}\right),
$$

but

$$
\operatorname{vol}_{3}\left(B_{r}\right)<\operatorname{vol}_{3}\left(B_{R}\right)
$$

\section{REFERENCES}

[B] K. Ball, Shadows of convex bodies, Trans. Amer. Math. Soc. 327 (1991) , 891901.

[G] R. J. Gardner, Geometric Tomography, Cambridge University Press, Cambridge 1995 .

[GKS] R. J. Gardner, A. Koldobsky, T. Schlumprecht, An analytic solution to the Busemann-Petty problem on sections of convex bodies, Annals of Math. 149 (1999), 691-703.

[GZ] P. GOODEy AND G. Zhang, Inequalities between projection functions of convex bodies, Amer. J. Math. 120 (1998), 345-367.

[K1] A. Koldobsky, A generalization of the Busemann-Petty problem on sections of convex bodies, Israel J. Math. 110 (1999), 75-91.

[K2] A. Koldobsky, Comparison of volumes by means of the areas of central sections, Adv. in Appl. Math. 33 (2004), no. 4, 728-732.

[K3] A. Koldobsky, Fourier Analysis in Convex Geometry, Mathematical Surveys and Monographs, American Mathematical Society, Providence RI, 2005.

[KKYY] N. J. Kalton, A. Koldobsky, V. Yaskin and M. Yaskina, The geometry of $L_{0}$, Canadian J. Math., to appear. http://arxiv.org/abs/math/0412371

[KRZ1] A. Koldobsky, D. Ryabogin And A. Zvavitch, Projections of convex bodies and the Fourier transform, Israel J. Math. 139 (2004), 361-380.

[KRZ2] A. Koldobsky, D. Ryabogin And A. Zvavitch, Unified Fourier analytic approach to the volume of projections and sections of convex bodies, Fourier Analysis and Convexity, (Editors: L. Brandolini, L. Cozani, A. Iosevich and G. Travaglini), Birkhauser 2004, 119-131.

[KYY] A. Koldobsky, V. Yaskin and M. Yaskina, Modified Busemann-Petty problem on sections on convex bodies, Israel J. Math., 154 (2006), 191-208.

[P] C. M. Petty, Projection bodies, Proc. Coll. Convexity (Copenhagen 1965), Kobenhavns Univ. Mat. Inst., 234-241.

[RZ] D. Ryabogin, A. Zvavitch, The Fourier transform and Firey projections of convex bodies, Indiana Univ. Math. J. 53 (2004), no. 3, 667-682.

[S1] R. SchneIDeR, Zur einem Problem von Shephard über die Projektionen konvexer Körper, Math. Z. 101 (1967), 71-82.

[S2] R. SChneIDER, Convex Bodies: the Brunn-Minkowski Theory, Cambridge University Press, Cambridge, 1993.

[YY] V. YASKIn AND M. YASKInA, Centroid bodies and comparison of volumes, Indiana University Math. J., 55 No. 3 (2006), 1175-1194.

V. Yaskin, Department of Mathematics, University of Oklahoma, Norman, OK 73019 , USA.

E-mail address: vyaskin@math.ou.edu 